Marquette University

e-Publications@Marquette

Mechanical Engineering Faculty Research and

Publications

Mechanical Engineering, Department of

$1-1-2006$

\title{
A Mass-Spring-Damper Model of a Bouncing Ball
}

Mark L. Nagurka

Marquette University, mark.nagurka@marquette.edu

Shuguang Huang

Marquette University, shuguang.huang@marquette.edu

Published version. International Journal of Engineering Education, Vol. 22, No. 2 (2006): 393-401.

Permalink. (C) 2006 Tempus Publications. Used with permission. 


\title{
A Mass-Spring-Damper Model of a Bouncing Ball*
}

\author{
MARK NAGURKA and SHUGUANG HUANG \\ Department of Mechanical and Industrial Engineering, Marquette University, Milwaukee, WI 53201, USA. \\ E-mail: mark.nagurka@marquette.edu,shuguang.huang@marquette.edu
}

\begin{abstract}
The mechanical properties of a vertically dropped ball, represented by an equivalent mass-springdamper model, are shown to be related to impact parameters. In particular, the paper develops relationships connecting the mass, stiffness and damping of a linear ball model to the coefficient of restitution and the contact time of the ball with the surface during one bounce. The paper also shows that the ball model parameters are functions of quantities readily determined in an experiment: (i) the height from which the ball is dropped from rest, (ii) the number of bounces, and (iii) the time elapsing between dropping the ball and the ball coming to rest. For a ball with significant bounce, approximate expressions are derived for the model parameters as well as for the natural frequency and damping ratio. Results from numerical and experimental studies of a bouncing ping-pong ball are presented.
\end{abstract}

Keywords: mechanics; dynamics; mass-spring damper model; coefficient of restitution

\section{INTRODUCTION}

THE BOUNCING BEHAVIOR of a dropped ball is a classic problem studied in the literature [1-8] and treated in textbooks of physics and dynamics that address the subject of impact. Many of these books also present, but in a separate section, the concept of mass, stiffness, and damping as the three elemental properties of a mechanical dynamic system. The behavior of such a system is represented by models comprised of series and parallel arrangements of masses, springs, and dampers, generally taken as linear elements. However, the books and literature do not connect the mechanical 'primitives' of mass, stiffness and damping to impact-related properties such as the coefficient of restitution, a measure of rebound behavior in a collision.

This paper develops this connection for a particular system, namely, a bouncing ball represented by a linear mass-spring-damper model. It is shown that the properties of the ball model can be related to the coefficient of restitution and the bounce contact time. Furthermore, assuming constant properties, it is shown that the coefficient of restitution can be related to quantities easily obtained in an experiment, namely, the height from which the ball is dropped from rest, the total number of bounces, and the total bounce time. For a ball with significant bounce, such as a ping-pong ball, approximate expressions for the properties are derived. The analytical findings are used to predict model properties of a ping-pong ball.

\footnotetext{
* Accepted 30 September 2005.
}

\section{Motivation}

A challenging and meaningful part of engineering education is the development of system models that reflect significant features of physical behavior. In many courses, classical models are presented, at times with limited motivation. Many undergraduate engineering textbooks present the same material in the same sequence, further stifling the excitement and creativity of developing models based on physical insight and simplifying assumptions. This paper is an attempt to take a fresh look at two topics that are well established and related but not connected in the typical presentation. The hope is that the student is afforded an opportunity to learn more about both topics by building the connection.

The physical properties of a real ball can be represented by its mass-spring-damping characteristics. These characteristics are introduced in courses such as system dynamics and fundamentals of vibrations. In these courses as well as classical dynamics courses, the topic of impact of bodies is often presented, but the connection between the physics of collisions and the equivalent mechanical properties is generally missed. The natural question is whether one can determine the linear stiffness and viscous damping of a bounced ball based on its impact behavior, essentially replacing the ball by an equivalent mass-springdamper system. The development presented here is based on a linear model that only partially captures the true physics. However, the intent is to prompt engineering students to synthesize material and better appreciate and question assumptions inherent in the classical derivations, with the ultimate aim being an enhanced understanding of fundamentals. This paper does not 
present a definitive advanced model, but rather the simplest model for determining the equivalent mechanical properties of the ball based on parameters readily available experimentally.

\section{MASS-SPRING-DAMPER MODEL}

The behavior of a vertically dropped ball is studied using the model illustrated in Fig. 1, where the ball is represented by its mass $m$, viscous damping $c$, and linear stiffness $k$. When the ball is not in contact with the ground, the equation of motion, assuming no aerodynamic drag, can be written simply as:

$$
m \ddot{x}=-m g,
$$

where $x$ is measured vertically up to the ball's center of mass with $x=0$ corresponding to initial contact, i.e., when the ball just contacts the ground with no deformation. The initial conditions are $x(0)=h_{0}$ and $\dot{x}(0)=0$ for a ball released from rest from height $h_{0}$. The solution of this simple problem appears in physics and mechanics textbooks, leading to the classical results of vertical projectile motion.

When the ball is in contact with the ground, deformation and restitution occur. The equation of motion is then:

$$
m \ddot{x}+c \dot{x}+k x=-m g
$$

with the initial conditions of $x(0)=0$ and $\dot{x}(0)=-v_{0}$ where $v_{0}$ is the velocity of the ball just prior to contact with the ground. Integrating Equation (2) gives:

$$
\begin{aligned}
x(t)= & {\left[\frac{c g-2 k v_{0}}{2 k \omega_{d}} \sin \omega_{d} t+\frac{m g}{k} \cos \omega_{d} t\right] } \\
& \times \exp \left(-\frac{c}{2 m} t\right)-\frac{m g}{k}
\end{aligned}
$$

where the damped natural frequency, $\omega_{d}$, is:

$$
\omega_{d}=\frac{1}{2 m} \sqrt{4 k m-c^{2}}
$$

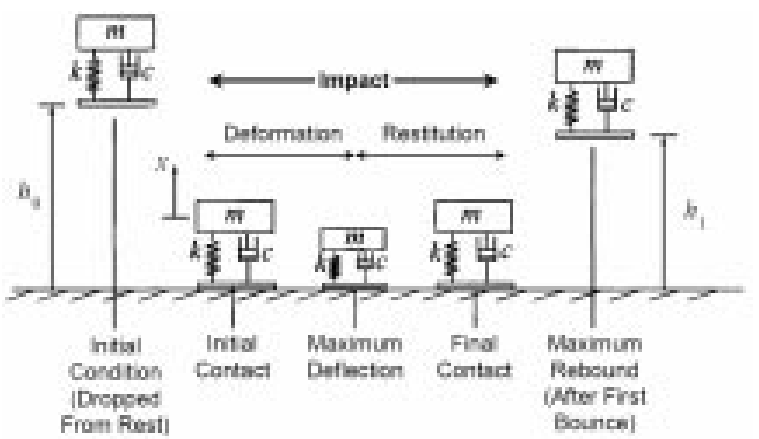

Fig. 1. A mass-spring-damper model of a ball showing impact phases at the first bounce. and $\exp (\beta)=e^{\beta}$, i.e., the base of the natural logarithm raised to the power $\beta$.

Equation (3) gives the motion of the ball during contact with the ground and applies only when $x(t) \leq 0$. Bounce behavior, involving deformation, restitution, and then rebound, requires an underdamped solution for which $\omega_{d}>0$ or $\left(4 \mathrm{~km}-\mathrm{c}^{2}\right)>0$. The 'steady' or rest solution, applying after the bounces have died out, can be obtained by setting $t \rightarrow \infty$ in Equation (3). The equilibrium position is:

$$
x^{*}=-\frac{m g}{k},
$$

and when $|x| \leq\left|x^{*}\right|$ there will be no further bounces. It follows that the number of bounces is finite.

\section{Contact time at first bounce}

The contact time, $\Delta T$, at the first bounce, shown in exaggerated view in Fig. 2, is the time from when the ball reaches $x=0$ after being dropped to the time it first comes back to $x=0$. Mathematically, the contact time is the first finite solution of the equation $x(\Delta T)=0$, i.e., from Equation (3) it is the minimum non-zero solution of:

$$
\begin{gathered}
{\left[\frac{c g-2 k v_{0}}{2 k \omega_{d}} \sin \left(\omega_{d} \Delta T\right)+\frac{m g}{k} \cos \left(\omega_{d} \Delta T\right)\right]} \\
\times \exp \left(-\frac{c \Delta T}{2 m}\right)-\frac{m g}{k}=0
\end{gathered}
$$

which in general has multiple solutions.

Equation (6) is difficult to solve analytically. A solution can be found numerically, or alternatively an approximate solution can be obtained by first writing Equation (3) in the rearranged form:

$$
\begin{aligned}
x(t)= & -\frac{v_{0}}{\omega_{d}} \exp \left(-\frac{c}{2 m} t\right) \sin \omega_{d} t-\frac{m g}{k} \\
& \times\left[1-\exp \left(-\frac{c}{2 m} t\right)\left(\cos \omega_{d} t+\frac{c}{2 m \omega_{d}} \sin \omega_{d} t\right)\right] .
\end{aligned}
$$

The maximum magnitude of the first term on the right-hand side, $v_{0} / \omega_{d}$, is the dynamic deformation due to the impact for the incoming velocity $v_{0}$; the

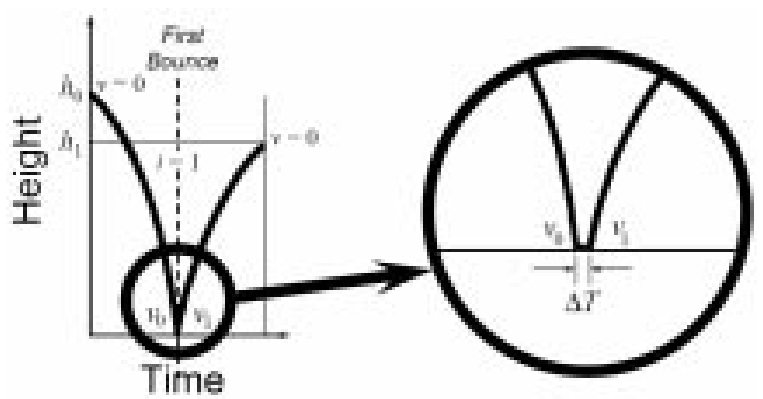

Fig. 2. Height versus time showing exaggerated view at first bounce. 
maximum magnitude of the second term, $m g / k$, is the static deformation due to the weight. Assuming $m g / k \ll\left|v_{0} / \omega_{d}\right|$, which is reasonable for a bouncing ball such as a ping-pong ball, the second term on the right-hand side in Equation (7) can be neglected and $x(t)$ can be approximated as:

$$
x(t)=-\frac{v_{0}}{\omega_{d}} \exp \left(-\frac{c}{2 m} t\right) \sin \omega_{d} t
$$

The contact time, $\Delta T$, can be found as the minimum nonzero solution of Equation (8) set equal to zero giving:

$$
\Delta T=\frac{\pi}{\omega_{d}}
$$

where $\omega_{d}$ is specified by Equation (4). Equation (9) represents an approximate solution for the contact time at the first bounce.

\section{Stiffness and damping at first bounce}

The ball stiffness, $k$, and damping, $c$, properties can be related to the contact time, $\Delta T$, and the coefficient of restitution, $\varepsilon$, where:

$$
\varepsilon=\left|\frac{\dot{x}(\Delta T)}{\dot{x}(0)}\right|
$$

is the ratio of speed of separation to speed of approach at the first bounce. The coefficient of restitution can be shown to be a measure of the kinetic energy lost in the collision. In a perfectly elastic collision, there is no loss and $\varepsilon=1$. In an inelastic collision, $0<\varepsilon<1$, some kinetic energy is transformed into deformation of the material, heat, sound, and other forms of energy, and is therefore unavailable. This loss is represented in the model by damping, $c$.

The denominator of Equation (10) is simply the speed of the ball prior to the first contact, $v_{0}$, and the numerator is the rebound or post-impact speed of the ball, $v_{1}$. The latter can be found by differentiating Equation (7) and imposing the assumption $m g / k \ll\left|v_{0} / \omega_{d}\right|$ or alternatively differentiating Equation (8) directly to give:

$$
\begin{aligned}
\dot{x}(t)= & \frac{c v_{0}}{2 m \omega_{d}} \exp \left(-\frac{c}{2 m} t\right) \sin \omega_{d} t \\
& -v_{0} \exp \left(-\frac{c}{2 m} t\right) \cos \omega_{d} t
\end{aligned}
$$

and then substituting $t=\Delta T$ with Equation (9) to give the rebound speed:

$$
v_{1}=\dot{x}(\Delta T)=v_{0} \exp \left(-\frac{c \Delta T}{2 m}\right) .
$$

From Equation (10), the coefficient of restitution can then be written simply as:

$$
\varepsilon=\exp \left(-\frac{c \Delta T}{2 m}\right) .
$$

For fixed $m$ and $c$, the shorter the contact time,
$\Delta T$, the larger the coefficient of restitution, $\varepsilon$, and vice-versa.

By manipulating Equations (4), (9), and (13), the stiffness, $k$, can be expressed as:

$$
k=m\left(\frac{\pi}{\Delta T}\right)^{2}\left[1+\left(\frac{\ln \varepsilon}{\pi}\right)^{2}\right],
$$

and by rearranging Equation (13) the viscous damping, $c$, can be written as:

$$
c=-\frac{2 m}{\Delta T} \ln \varepsilon .
$$

From Equations (14) and (15), a shorter contact time, $\Delta T$, corresponds to both a higher stiffness, $k$, and damping, $c$. In addition, as $\varepsilon$ increases, there is a negligible change in $k$ and a reduction in $c$.

\section{Natural frequency and damping ratio at first} bounce

The undamped natural frequency, $\omega_{n}=\sqrt{k / m}$, can be written from Equation (14) as:

$$
\omega_{n}=\frac{\pi}{\Delta T} \sqrt{1+\left(\frac{\ln \varepsilon}{\pi}\right)^{2}}
$$

and is a function of the contact time, $\Delta T$, and the coefficient of restitution, $\varepsilon$. The damping ratio, $\zeta$ :

$$
\zeta=\sqrt{1-\frac{\omega_{d}^{2}}{\omega_{n}^{2}}}=\frac{c}{2 \sqrt{k m}},
$$

can be found by substituting Equations (9) and (16) or Equations (14) and (15) giving:

$$
\zeta=\frac{-\ln \varepsilon}{\pi}\left[1+\left(\frac{\ln \varepsilon}{\pi}\right)^{2}\right]^{-1 / 2} .
$$

The damping ratio depends solely on the coefficient of restitution, $\varepsilon$.

\section{Coefficient of restitution related to total time and number of bounces}

In the following, it is shown that the coefficient of restitution, $\varepsilon$, can be related to the initial height from which the ball is dropped, $h_{0}$, the total number of bounces of the ball, $n$, and the total time, $T_{\text {total }}$, that elapses from when the ball is dropped until it comes to rest. These parameters, indicated in the bounce history diagram of Fig. 3, can easily be determined in an experiment.

It is assumed that $k, c, m$, and $\varepsilon$ are constant (independent of the velocity). With $n$ and $T_{\text {total }}$ assumed known, $\Delta T$ and $\varepsilon$, and thus $k$ and $c$, can be determined under the assumptions of constant $\Delta T$ and $\varepsilon$ for all bounces and negligible aerodynamic drag.

The total time, $T_{\text {total }}$, is comprised of flight times and contact times:

$$
T_{\text {total }}=T_{\text {flight }}+T_{\text {contact }}
$$




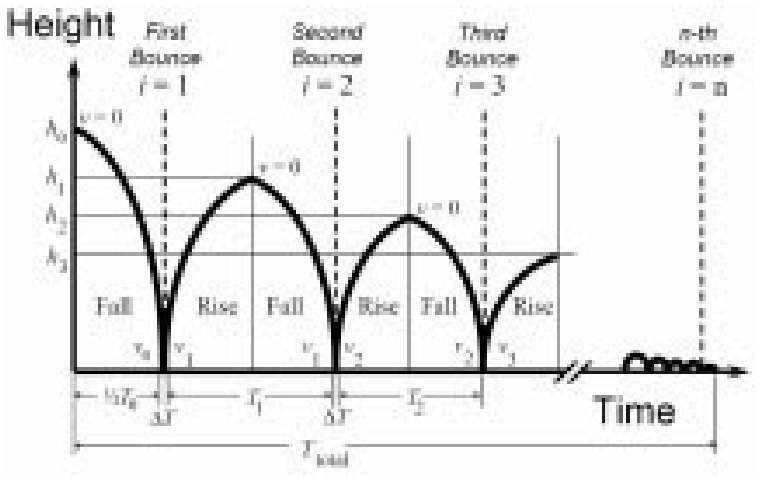

Fig. 3. Bounce history showing height versus time.

The sum of the flight times, $T_{\text {flight }}$, is:

$$
T_{\text {flight }}=\frac{1}{2} T_{0}+\sum_{i=1}^{n} T_{i},
$$

where the time to the first impact is $\frac{1}{2} T_{0}$, as indicated in Fig. 3. Noting that the flight time for the $i$ th bounce $(i \geq 1)$ can be written as:

$$
T_{i}=\varepsilon T_{i-1}
$$

since $v_{i}=\varepsilon v_{i-1}$ and $v_{i}=\frac{1}{2} g T_{i}$, the sum of the flight times for $n$ bounces can be calculated using Equation (19) as:

$$
\begin{aligned}
T_{\text {flight }} & =\frac{1}{2} T_{0}+T_{1}\left(1+\varepsilon+\ldots+\varepsilon^{n-1}\right) \\
& =\frac{1}{2} T_{0}+T_{0} \varepsilon\left(\frac{1-\varepsilon^{n-1}}{1-\varepsilon}\right)
\end{aligned}
$$

or

$$
T_{\text {flight }}=\frac{1}{2} T_{0}\left(\frac{1+\varepsilon-2 \varepsilon^{n}}{1-\varepsilon}\right) .
$$

Since the time to the first impact:

$$
\frac{1}{2} T_{0}=\sqrt{\frac{2 h_{0}}{g}},
$$

where $h_{0}$ is the initial height of the ball, the total flight time for $n$ bounces can alternatively be expressed as:

$$
T_{\text {flight }}=\sqrt{\frac{2 h_{0}}{g}}\left(\frac{1+\varepsilon-2 \varepsilon^{n}}{1-\varepsilon}\right) .
$$

In Equation (18) the sum of the contact times, $T_{\text {contact }}$, can be written:

$$
T_{\text {contact }}=n \Delta T,
$$

assuming the contact time, $\Delta T$, at each bounce is identical. It is possible to develop an expression for $\Delta T$ in terms of $\varepsilon, n$, and $h_{0}$. For the $i$-th bounce, the height the ball can reach is:

$$
h_{i}=\varepsilon^{2 i} h_{0} .
$$

Equation (25) can be derived from $v_{i}=\varepsilon v_{i-1}=\varepsilon^{i} v_{0}$ and $v_{i}=\sqrt{2 g h_{i}}$. For the ball to come to rest:

$$
h_{n}=\varepsilon^{2 n} h_{0} \leq \frac{m g}{k}
$$

where the upper limit is given by the equilibrium position of Equation (5). Substituting Equation (14) into the equality of Equation (26) and rearranging gives an expression for $\Delta T$ :

$$
\Delta T=\pi \varepsilon^{n} \sqrt{\frac{h_{0}}{g}\left[1+\left(\frac{\ln \varepsilon}{\pi}\right)^{2}\right] .}
$$

Substituting Equations (23), (24), and (27) into (18) gives:

$$
\begin{aligned}
T_{\text {total }}= & \sqrt{\frac{h_{0}}{g}} \\
& \times\left[\sqrt{2}\left(\frac{1+\varepsilon-2 \varepsilon^{n}}{1-\varepsilon}\right)+\pi n \varepsilon^{n} \sqrt{1+\left(\frac{\ln \varepsilon}{\pi}\right)^{2}}\right] .
\end{aligned}
$$

Equation (28) can be viewed as a single equation for unknown $\varepsilon$ in terms of $h_{0}, n$, and $T_{\text {total }}$. Alternatively, it can be written in nondimensional form:

$$
\tau=\left(\frac{1+\varepsilon-2 \varepsilon^{n}}{1-\varepsilon}\right)+\frac{\sqrt{2}}{2} \pi n \varepsilon^{n} \sqrt{1+\left(\frac{\ln \varepsilon}{\pi}\right)^{2}},
$$

where

$$
\tau=\frac{T_{\text {total }}}{\frac{1}{2} T_{0}}
$$

is the total time divided by the time to the first impact. Similarly, the contact time of Equation (27) can be presented in nondimensional form:

$$
\Delta \tau=\frac{\sqrt{2}}{2} \pi \varepsilon^{n} \sqrt{1+\left(\frac{\ln \varepsilon}{\pi}\right)^{2}},
$$

where

$$
\Delta \tau=\frac{\Delta T}{\frac{1}{2} T_{0}},
$$

is the contact time divided by the time to the first impact.

\section{Approximate relationships}

It is possible to develop simplified approximate relationships for the case of $|(\ln \varepsilon) / \pi| \ll 1$, which is representative of a ball with significant bounce, such as a ping-pong ball. For example, a ratio of $|(\ln \varepsilon) / \pi|$ of 0.1 or smaller corresponds to $0.73<\varepsilon<1$. 
For this case (for which $[(\ln \varepsilon) / \pi]^{2}$ is 0.01 or smaller), the stiffness, $k$, given by Equation (14) can be approximated as:

$$
k \cong m\left(\frac{\pi}{\Delta T}\right)^{2} .
$$

Alternatively, it could be expressed as an approximation of Equation (16):

$$
\omega_{n} \cong \frac{\pi}{\Delta T},
$$

which itself is an approximation of Equation (9). Thus, approximately the contact time at a single bounce is simply $\pi$ times the inverse of the undamped natural frequency. The contact time can also be approximated from Equation (27) as:

$$
\Delta T \cong \pi \varepsilon^{n} \sqrt{\frac{h_{0}}{g}},
$$

or, in nondimensional form, from Equation (31) as:

$$
\Delta \tau \cong \frac{\sqrt{2}}{2} \pi \varepsilon^{n}
$$

From Equation (17), the damping ratio, $\zeta$, for the case of higher values of $\varepsilon$ is:

$$
\zeta \cong \frac{-\ln \varepsilon}{\pi}
$$

providing a simple direct connection between the damping ratio and the coefficient of restitution.

For larger $n$, Equation (28) can be simplified to:

$$
T_{\text {total }} \cong \sqrt{\frac{2 h_{0}}{g}}\left(\frac{1+\varepsilon}{1-\varepsilon}\right),
$$

which expresses $T_{\text {total }}$ as the product of a factor depending only on $\varepsilon$ (in parentheses) and the time to the first impact, $\sqrt{2 h_{0} / g}$, or in nondimensional form:

$$
\tau \cong \frac{1+\varepsilon}{1-\varepsilon}
$$

Equations (38) and (39), which neglect the contact times, do not depend on $n$, and can be rearranged to find a simple equation for $\varepsilon$ in terms of $T_{\text {total }}$ for a given $h_{0}$.

Finally, it is possible to arrive at expressions for the stiffness/mass and damping/mass ratios in terms of the coefficient of restitution and number of bounces. From Equations (14) and (27),

$$
\frac{k}{m}=\frac{g}{h_{0}} \varepsilon^{-2 n},
$$

and from Equations (15) and (35):

$$
\frac{c}{m} \cong-\frac{2}{\pi} \sqrt{\frac{g}{h_{0}}} \varepsilon^{-n} \ln \varepsilon
$$

where, from Equation (39), the coefficient of restitution, $\varepsilon$, can be written:

$$
\varepsilon \cong \frac{\tau-1}{\tau+1} .
$$

\section{NUMERICAL AND EXPERIMENTAL STUDIES}

A ping-pong ball (new Harvard, one-star) was dropped from rest from a measured initial height of $30.5 \mathrm{~cm}$ onto a laboratory bench with a butcherblock top. The acoustic signals accompanying the ball-table impacts were recorded using a microphone attached to the sound card of a PC. The method follows the procedure described in [5]. From the temporal history of the bounce sounds of successive impacts, the total number of bounces was determined to be $n=64$ and the total bounce time was determined to be $T_{\text {total }}=6.2 \mathrm{~s}$. The mass of the ball used in the experiment was measured to be $m=2.70 \mathrm{~g}$.

The sequence of steps to determine the model parameters is as follows:

1. With known initial height $h_{0}$, number of bounces, $n$, and total time $T_{\text {total }}$, determine the coefficient of restitution, $\varepsilon$, from Equation (28).

2. With known $h_{0}$ and $n$ and calculated $\varepsilon$, determine the contact time, $\Delta T$, from Equation (27).

3. With known $m$ and calculated $\varepsilon$ and $\Delta T$, determine the stiffness, $k$, and damping, $c$, from Equations (14) and (15), respectively.

\section{Predicted coefficient of restitution}

The coefficient of restitution, $\varepsilon$, can be found from Equation (28) given $h_{0}, n$, and $T_{\text {total }}$. The relationship is shown in Fig. 4 for the case of $h_{0}=30.5 \mathrm{~cm}$ and indicates that the total time does not depend significantly on the number of bounces, especially for a large number of bounces where the asymptotic values are given by the approximation of Equation (38).

Figure 4 provides a means to identify by inspection the coefficient of restitution. For $T_{\text {total }}=6.2 \mathrm{~s}$ and $n=64$, the coefficient of restitution is $\varepsilon=0.92$. This value is also predicted from the approximate Equation (38), or equivalently from Equation (42) with $\tau=24.9$ from Equations (22) and (30). (Note that the time to the first impact is 0.249 s from Equation (22).)

\section{Predicted contact time}

With the coefficient of restitution determined, the contact time, $\Delta T$, at a single bounce can be found from Equation (27) or the approximation of Equation (35). Equation (27) is shown graphically in Fig. 5, from which $\Delta T$ can be determined by inspection given the values of $n$ and $\varepsilon$. In the experiment, for $n=64$ and $\varepsilon=0.92$, the predicted contact time $\Delta T=3.2 \mathrm{~ms}$. 


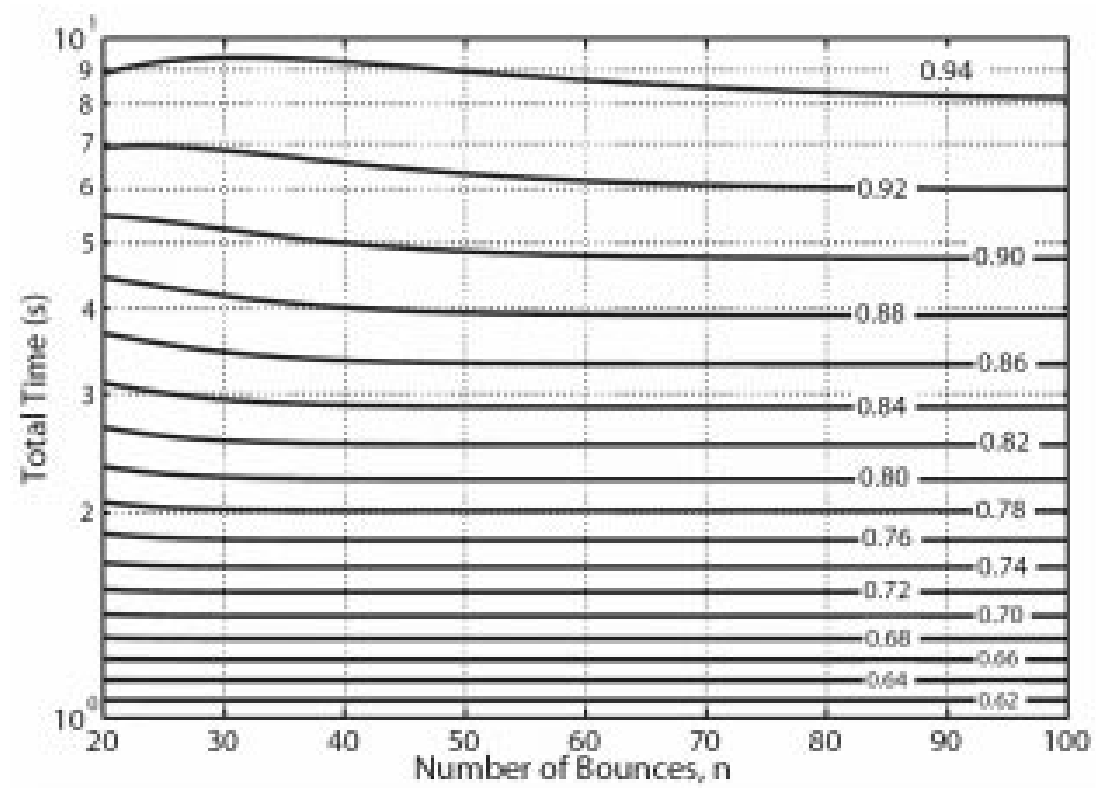

Fig. 4. Total time, $T_{\text {total }}$, as a function of number of bounces, $n$, and coefficient of restitution, $\varepsilon$ (marked on curve), from Equation (28) for a drop height, $h_{0}$, of $30.5 \mathrm{~cm}$.

\section{Predicted stiffness and damping}

Given values for the coefficient of restitution and contact time, the linear stiffness and viscous damping of an equivalent mass-spring-damper model of the ball can be determined. An expression for the stiffness is given in Equation (14) and in simplified approximate form in Equation (33). Figure 6 graphically depicts these relationships in terms of $k / m$ as a function of $\varepsilon$ for different values of $\Delta T$. The approximate equation (33) provides a highly accurate prediction of $k$ from Equation (14), showing only slight deviation at smaller values of $\varepsilon$. The damping coefficient $c$, given by Equation (15), is plotted in Fig. 7 in terms of $c / m$ as a function of $\varepsilon$ and $\Delta T$, showing clear dependence on both.
For the case of the ping-pong ball dropped from an initial height of $30.5 \mathrm{~cm}$ and with $\Delta T$ determined to be $3.2 \mathrm{~ms}, k / m=9.6 \times 10^{5} \mathrm{~s}^{-2}$. For $m=2.7 \mathrm{~g}$, the stiffness $k=2.6 \mathrm{~N} / \mathrm{mm}$ (or $\mathrm{kPa}$ ). For $\varepsilon=0.92$ and $\Delta T=3.2 \mathrm{~ms}, c / m=50 \mathrm{~s}^{-1}$, and for $m=2.7 \mathrm{~g}$ the damping coefficient $c=0.135 \mathrm{~N}-\mathrm{s} / \mathrm{m}$.

\section{Predicted natural frequency and damping ratio}

From Equation (16) or the approximation from a rearrangement of Equation (34) it is possible to find the natural frequency, $\omega_{n}$. For $\Delta T=3.2 \mathrm{~ms}$ and $\varepsilon=0.92, \omega_{n}=980 \mathrm{rad} / \mathrm{s}$ or $155 \mathrm{~Hz}$. From Equation (17) or the approximation of Equation (37) it is possible to predict the damping ratio, $\zeta$.

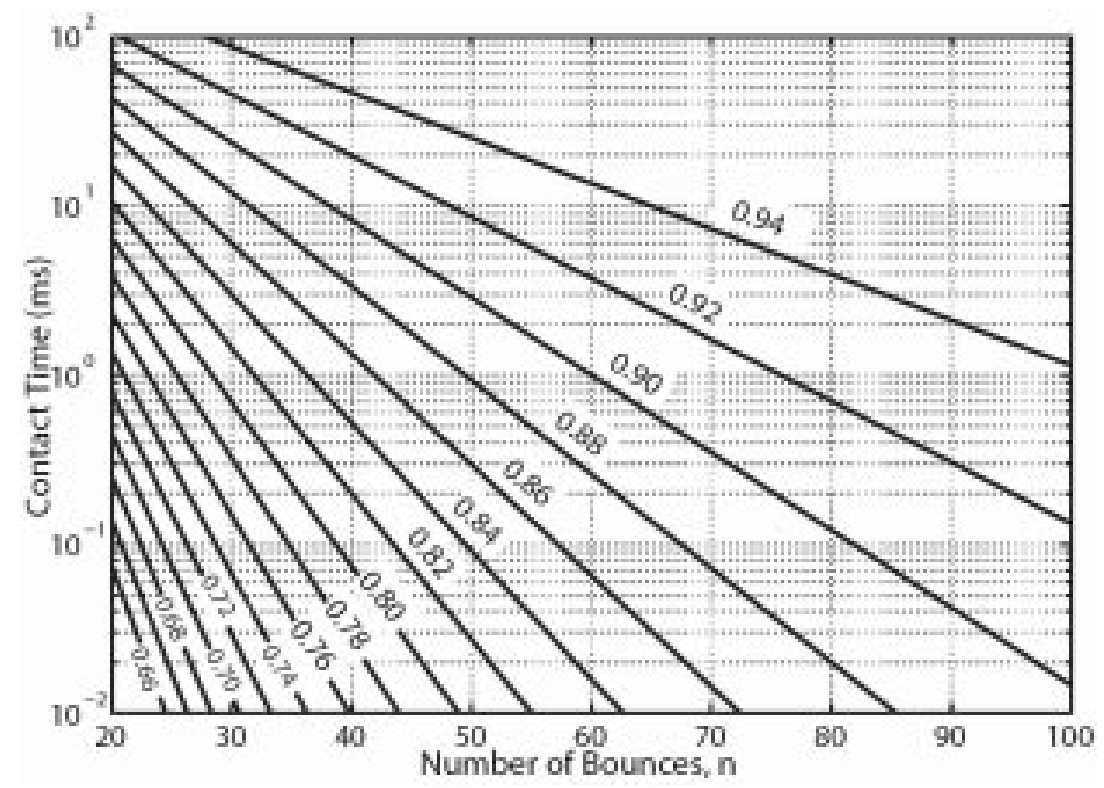

Fig. 5. Contact time, $\Delta T$, at a single bounce as a function of number of bounces, $n$, and coefficient of restitution, $\varepsilon$ (marked on curve), from Equation (27), and from approximation from Equation (35), for a drop height, $h_{0}$, of $30.5 \mathrm{~cm}$. 


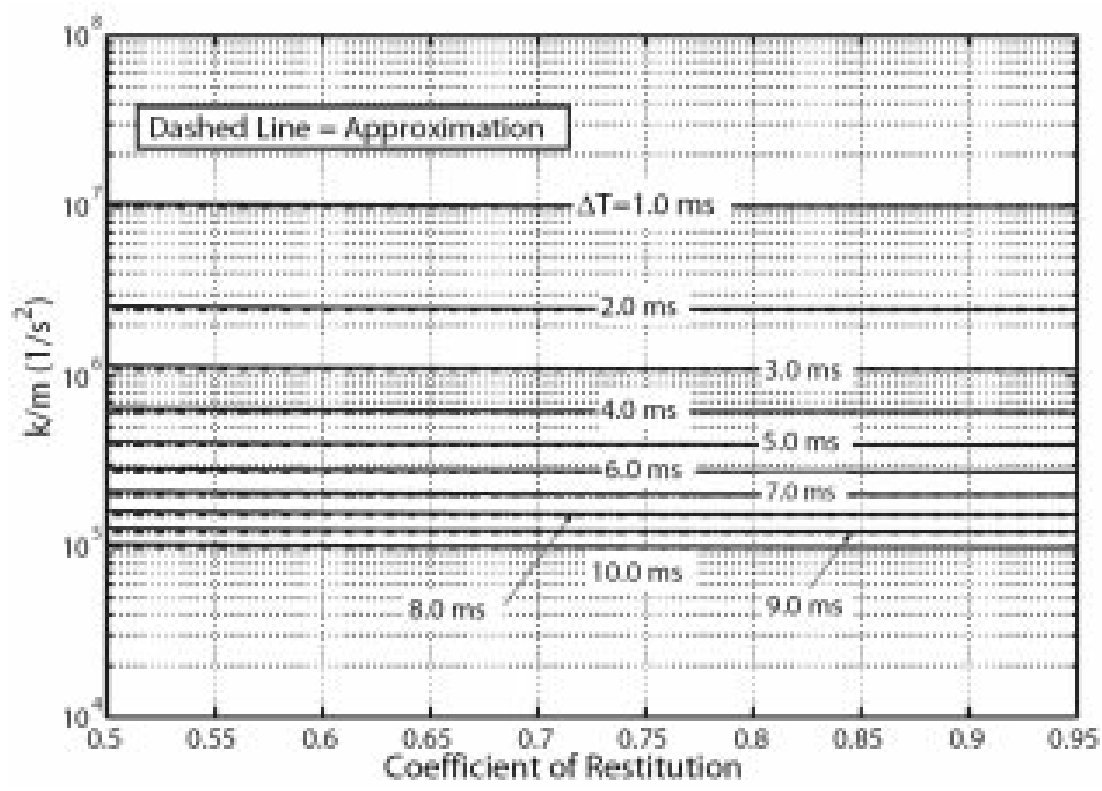

Fig. 6. Stiffness divided by mass, $k / m$, as a function of coefficient of restitution, $\varepsilon$, and contact time, $\Delta T$, from Equation (14) and for approximation from Equation (33).

For $\varepsilon=0.92, \zeta=0.026$, indicating a very lightly underdamped system.

\section{DISCUSSION}

The vertical bounce history of a ball, dropped from rest, provides clues that can be used to determine an equivalent mechanical model of the ball. In the formulation, the ball is modeled as having constant and linear mass, stiffness, and damping properties ( $m, k$, and $c$, respectively), which are related to key features of the bounce behavior (namely, $h_{0}, n$, and $T_{\text {total }}$ ). This fixed mass-spring-damper model precludes accounting for nonlinear stiffness and friction effects in the impact. For example, the model cannot represent Hertzian force-displacement characteristics (e.g., force related to displacement to the $2 / 3$ power) nor Coulomb-type damping and hysteretic losses at the interface that are presumed to act.

In addition to fixed model parameters for the ball, it is assumed that at all impacts there is a constant coefficient of restitution, $\varepsilon$. A consequence of assuming that the parameters $m, k, c$ and $\varepsilon$ are constant is that contact time, $\Delta T$, at each bounce is constant. A more advanced model, incorporating nonlinear elements and/or a changing

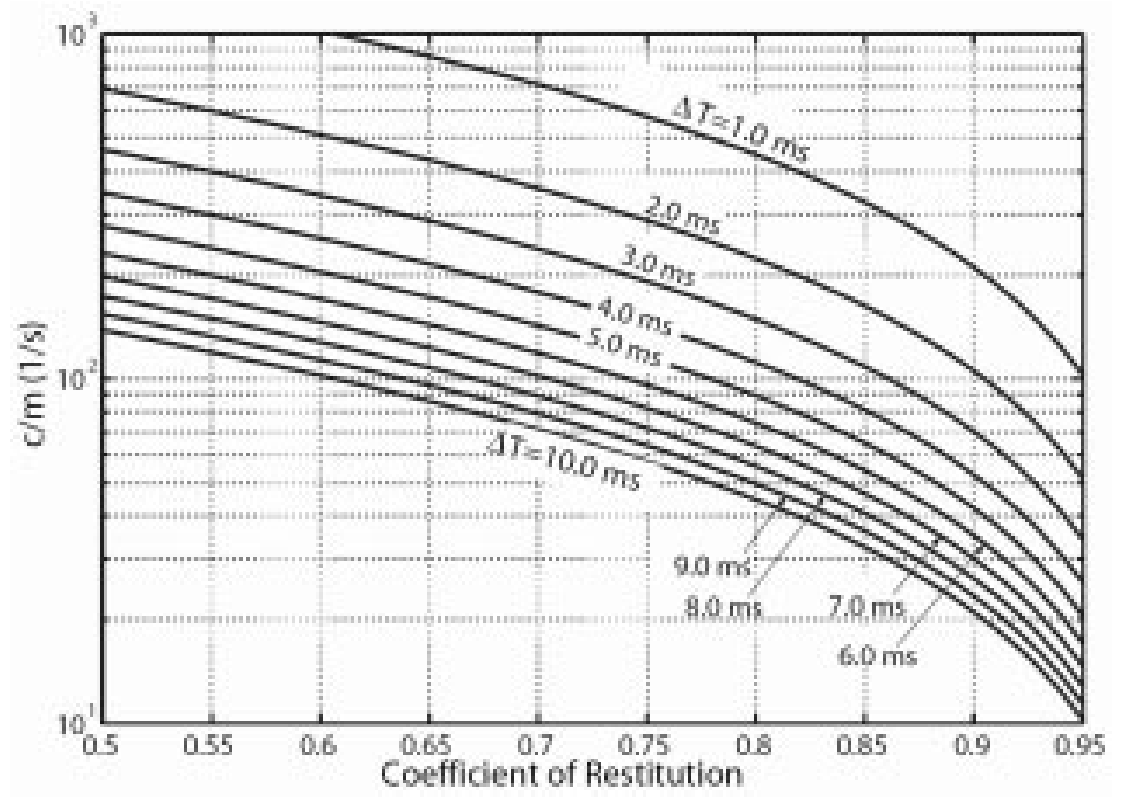

Fig. 7. Damping coefficient divided by mass, $c / m$, as a function of coefficient of restitution, $\varepsilon$, and contact time, $\Delta T$, from Equation (15). 
coefficient of restitution, would be needed to account for a contact time that decreases with each bounce.

The total time from when the ball is dropped until when it comes to rest is comprised of flight times and contact times. Although the sum of the contact times $\left(T_{\text {contact }}\right)$ for all bounces is a small fraction of the sum of the flight times $\left(T_{\text {flight }}\right)$, it is included in the model. (Here, this fraction is approximately $3.5 \%$.) The analysis neglects aerodynamic effects, which occur in reality during the flight times. By not accounting for aerodynamic drag of the ball during flight, the approach gives a higher coefficient of restitution than otherwise would be predicted.

Several observations can be made:

- the larger the contact time $\Delta T$, the smaller the stiffness $k$ and the damping $c$;

- the larger the coefficient of restitution $\varepsilon$, the smaller the damping $c$;

- the coefficient of restitution $\varepsilon$ does not strongly influence the stiffness $k$;

- the larger the coefficient of restitution $\varepsilon$, the larger the total time;

- the number of bounces $n$ (for $n>0$ ) does not strongly influence the total time.

Finally, as an aside, it is noted that some people, including the sight-impaired, can gauge reasonably accurately the drop height of a ping-pong ball based on listening to the bounce time history. This ability may be a subconscious synthesis of knowing the total time and number of bounces.

\section{Pedagogy}

In the interest of motivating students to stretch beyond the rather rigid formalism of classical presentations, this paper bridges two distinct but clearly related topics. The analysis is limited by several questionable assumptions, such as fixed, linear properties of the ball, constant coefficient of restitution, constant contact time, and no aerodynamic drag. These assumptions and others, such as vertical motion only of a non-rotating ball, can each be addressed in more detail with discussion of their significance, and more advanced models can be developed. The analysis is suited to the level of an undergraduate engineering student.

\section{CLOSING}

This paper examines relationships linking linear mechanical model parameters, namely the mass, stiffness, and damping of a bouncing ball, with classical mechanical impact parameters, i.e., the coefficient of restitution and the time of contact between a ball and a surface. Under the assumption of no aerodynamic drag and constant coefficient of restitution for all bounces, the stiffness/ mass and damping/mass ratios, or alternatively the natural frequency and damping ratio, can be expressed explicitly in terms of the coefficient of restitution and time of contact. Ball properties are shown to be related to parameters easily found in an experiment, namely, the height from which the ball is dropped, the number of bounces, and the total bounce time. Also considered is the special case of bouncing balls with higher values of coefficient of restitution for which simple approximate expressions can be derived for model parameters. The results of an experimental test are used to provide predictions of the equivalent stiffness and damping, natural frequency and damping ratio, and coefficient of restitution for a bouncing pingpong ball.

\section{REFERENCES}

1. L. Flansburg and K. Hudnut, Dynamic Solutions for Linear Elastic Collisions, American J. Physics, 47, 1979, pp. 911-914.

2. E. Falcon, C. Laroche, S. Fauve and C. Costa, Behavior of one inelastic ball bouncing repeatedly off the ground, The European Physical Journal, 3, 1998, pp. 45-57.

3. L. Pauchard and S. Rica, Contact and compression of elastic spherical shells: the physics of a pingpong ball, Philosophical Magazine B, 78(2), 1998, pp. 225-233.

4. C. Cross, The Bounce of a Ball, American Journal of Physics, 67(3), 1999, pp. 222-227.

5. M. L. Nagurka, A simple dynamics experiment based on acoustic emission, Mechatronics, 12(2), 2002, pp. 229-239.

6. M. L. Nagurka, Aerodynamic effects in a dropped ping-pong ball experiment, Int. J. Eng. Educ., 19(4), 2003, pp. 623-630.

7. C. Wu, L. Li and C. Thornton, Rebound behavior of spheres for plastic impacts, Int. J. Eng. Educ., 28, 2003, pp. 929-946.

8. E. Kanso and P. Papadopoulos, Dynamics of pseudo-rigid ball impact on rigid foundation, Int. J. Non-Linear Mechanics, 39, 2004, pp. 299-309.

Mark Nagurka is an Associate Professor of Mechanical and Biomedical Engineering at Marquette University (Milwaukee, WI). He earned a Ph.D. in Mechanical Engineering from M.I.T. (Cambridge, MA), taught at Carnegie Mellon University (Pittsburgh, PA), and was a Senior Research Engineer at the Carnegie Mellon Research Institute (Pittsburgh, PA). Dr. Nagurka is a registered Professional Engineer in Wisconsin and Pennsylvania, a Fellow of the American Society of Mechanical Engineers (ASME), and a former Fulbright 
Scholar. His research interests include mechatronics, automation, control system design, human/machine interaction, and vehicle dynamics.

Shuguang Huang is a Research Assistant Professor in the Department of Mechanical and Industrial Engineering at Marquette University (Milwaukee WI). He received a BS degree in Mechanics and an MS degree in Theoretical Mechanics from Peking University (Beijing, China), earned a Ph.D. degree in Mechanical Engineering from Marquette University, and taught in the Department of Engineering Mechanics at Tsinghua University (Beijing, China). Dr. Huang is a member of IEEE. His general interests include classical mechanics, control and design of robotic systems. 